\title{
Determinación de lesiones encefálicas en canes mediante tomografía computarizada en Lima, Perú
}

\author{
Determination of brain injuries in dogs through computed \\ tomography in Lima, Peru
}

Claudia Ojeda L. ${ }^{1,2}$, Eben Salinas C. ${ }^{1,3}$

\section{Resumen}

El objetivo del presente estudio fue determinar lesiones encefálicas mediante tomografía computarizada (TC) en 71 canes que fueron sometidos al estudio encefálico por recomendación médica entre 2011 y 2015 en Lima, Perú. Los diagnósticos tomográficos fueron relacionados con el sexo, edad, tamaño y raza de cada paciente. Se identificaron 38 diagnósticos tomográficos positivos a lesión encefálica (53.5\%). El grupo etario positivo a lesión cerebral más frecuente fue el comprendido entre 1 y 7 años; así como el tamaño de perro mediano. Las lesiones diagnosticadas más frecuentes fueron dilatación ventricular $(29 \%, 11 / 38)$, neoformaciones encefálicas $(15.8 \%, 6 / 38)$ e hidrocefalia $(15.8 \%, 6 / 38)$. La dilatación ventricular se presentó con mayor frecuencia entre 1 y 7 años, en razas Maltés y Poodle, las neoformaciones cerebrales en canes mayores de 7 años y en la raza Labrador, en tanto que la hidrocefalia en canes entre 1 y 7 años, mayormente en Chihuahua y Pug.

Palabras clave: encéfalo; tomografía computarizada; dilatación ventricular; hidrocefalia; neoformación

\section{AbSTRaCT}

The aim of this study was to determine encephalic lesions by computed tomography (CT scan) in 71 dogs that were submitted to the encephalic study by medical recommendation between 2011 and 2015 in Lima, Peru. The tomographic diagnoses were related to the sex, age, size and breed of the patients. Thirty-eight positive tomographic

\footnotetext{
${ }^{1}$ Carrera de Medicina Veterinaria y Zootecnia, Universidad Científica del Sur, Lima, Perú

${ }^{2}$ E-mail: claudiaojedaluna@live.com

${ }^{3}$ E-mail: esalinasc@cientifica.edu.pe
}

Recibido: 8 de agosto de 2018

Aceptado para publicación: 18 de enero de 2019 
diagnoses of brain injury were identified (53.5\%). The most frequent positive age group to brain injury was between 1 and 7 years and in medium-size dogs. The most frequent diagnosed lesions were ventricular dilation $(29 \%, 11 / 38)$, brain neoformations $(15.8 \%, 6 /$ $38)$ and hydrocephalus $(15.8 \%, 6 / 38)$. Ventricular dilation occurred more frequently between 1 and 7 years and in Maltese and Poodle breeds, brain neoformations in dogs older than 7 years and in the Labrador breed, while hydrocephalus in dogs between 1 and 7 years old, mostly in Chihuahua and Pug.

Key words: brain; computerized tomography; ventricular dilatation; hydrocephalus; neoformation

\section{INTRODUCCIÓN}

La tomografía computarizada (TC) utiliza un haz de rayos $\mathrm{X}$ dirigido y de un grosor determinado, dependiente del tamaño de la estructura a estudiar, lo cual permite una adecuada diferenciación de tejidos blandos $\mathrm{y}$ morfologías adyacentes, en detalles de hasta un milímetro (Aguinaga et al., 2006). La TC puede brindar información respecto a diversas afecciones a nivel encefálico, utilizándose en medicina veterinaria para el diagnóstico de neoplasias intracraneales, identificación de masas de carácter no neoplásico o neoformaciones, quistes, parásitos, lesiones isquémicas y patologías de carácter inflamatorio focal o generalizadas (Calzado y Gelegins, 2010; Lorigados y Pinto, 2013; Przyborowska et al., 2013).

La mayoría de los informes de anormalidades halladas en exploraciones por TC a nivel encefálico se han centrado en el diagnóstico de tumores cerebrales (Snyder et al., 2006; Babicsak et al., 2011). Además, otro tipo de lesiones pueden ser diagnosticadas mediante la TC, como son las anormalidades congénitas, tipo hidrocefalia, agenesias, hipoplasias y diversas malformaciones (Calzado y Gelegins, 2010; Lorigados y Pinto, 2013). Así mismo, la TC puede ser usada para identificar áreas de hemorragia, edema, infarto tisular, hipertensión y diversos tipos de accidentes cerebro vasculares (Uscanga et al., 2005; Machado et al., 2007; Rodríguez et al., 2010; Lorigados y Pinto, 2013). Los cortes transversales producen una imagen bidimensional, sin superposición de estructuras, que permite una adecuada diferenciación con relación a la calota craneana (Machado et al., 2007; Calzado y Gelegins, 2010). Raurell et al. (2007) afirmó que la TC es la prueba diagnóstica más rápida para la identificación de la enfermedad vascular cerebral.

La TC es la modalidad más sensible para la evaluación de anormalidades óseas y en casos de traumatismo encéfalo craneano. La obtención de imágenes es rápida y la reconstrucción espacial de imágenes en 3D es posible con el software adecuado. Es posible evidenciar anormalidades radiológicas, incluso en animales con trauma ligero (Luján, 2007). La TC en estadios tempranos del trauma permite identificar al edema cerebral difuso, uno de los principales motivos de la evolución fatal del paciente traumatizado (Uscanga et al., 2005; Rodríguez et al., 2010). Cabe mencionar que la resonancia magnética (RM) puede estar contraindicada para la investigación del traumatismo encefalocraneano y de los pacientes de cuidados críticos, debido a la duración de la toma de la imagen y al hecho de que el fuerte campo magnético impide el uso de determinados dispositivos de soporte y monitorización del paciente. Es por eso que las fracturas del cráneo pueden ser identificadas con mayor facilidad mediante TC que con RM, por su mayor sensibilidad por el tejido óseo (Luján, 2007) 
El diagnóstico adecuado de las patologías encefálicas y síndromes de carácter neurológico permite instaurar un tratamiento oportuno que redunda en la salud y calidad de vida del paciente. Así mismo, el diagnóstico temprano permite al médico realizar maniobras o cirugías que se requieran para preservar la vida del paciente. Es ahí donde se resalta la importancia de conocer de manera más adecuada las diversas patologías a nivel encefálico, así como su correcta identificación imagenológica. El presente estudio se realiza con el objetivo de determinar las lesiones encefálicas mediante TC en una población de 71 canes durante un periodo de cuatro años (2011-2015). Además, se busca relacionar estos hallazgos con el sexo, edad, tamaño y raza de los pacientes.

\section{Materiales y Métodos}

El estudio incluyó 71 historias clínicas y sus respectivos informes de tomografía computarizada a nivel de encéfalo en el periodo entre 2011 y 2015 en clínicas veterinarias de Lima Metropolitana, Perú. Todos los informes tomográficos correspondieron a pacientes con afecciones neurológicas, evaluadas previamente en una consulta neurológica. Los pacientes fueron de ambos sexos, con edades entre los 0.2 y 15 años y de cuatro tamaños con relación a la raza de cada individuo (Cuadro 1).

En la evaluación tomográfica se tomó en cuenta la ubicación de la lesión (hemisferio cerebral o región cerebral afectada), descripción de la lesión y el diagnóstico tomográfico. Se tomaron en cuenta las características demográficas como edad, sexo, tamaño y raza. Las tomografías fueron realizadas en el Centro de Diagnóstico EMETAC, en Lima, Perú, con un tomógrafo de segunda generación Siemens Somaton Spirit Dual Slice CT Scanner (dos cortes).
Cuadro 1. Características de 71 canes sometidos a tomografía computarizada encefálica en Lima, Perú (2011-2015)

\begin{tabular}{lcc}
\hline Características & $\mathrm{n}$ & $\%$ \\
\hline Sexo & & \\
$\quad$ Machos & 39 & 54.9 \\
$\quad$ Hembras & 32 & 45.1 \\
Grupo etario (años) & & \\
$\quad=1$ & 14 & 19.7 \\
$1-7$ & 29 & 40.9 \\
$\quad>7$ & 28 & 39.4 \\
Raza/tamaño & & \\
$\quad$ Miniatura $(<5 \mathrm{~kg})$ & 8 & 11.3 \\
$\quad$ Pequeña $(6-10 \mathrm{~kg})$ & 24 & 33.8 \\
$\quad$ Mediana $(11-25 \mathrm{~kg})$ & 27 & 38.0 \\
$\quad$ Grande $(26-35 \mathrm{~kg})$ & 12 & 16.9 \\
\hline
\end{tabular}

En las variables de tipo cualitativo se identificó la frecuencia absoluta y relativa de los resultados para sus respectivos estratos y esta información se detalló en tablas de frecuencia.

\section{Resultados}

De los 71 diagnósticos tomográficos de canes sometidos a estudios encefálicos se identificaron 38 diagnósticos tomográficos positivos a lesión encefálica, representando en general una frecuencia de lesión diagnosticada por TC de $53.5 \%$.

Las lesiones más frecuentes en pacientes con diagnostico tomográfico fueron la dilatación ventricular $(29 \%, 11 / 38)$, neoformación encefálica $(15.8 \%, 6 / 38)$, hidrocefalia $(15.8 \%, 6 / 38)$, lesión isquémica $(10.5,4 / 38)$ e hipertensión endocraneana 
Cuadro 2. Diagnósticos tomográficos y lesiones asociadas más frecuentes en tomografías encefálicas de 38 canes en Lima, Perú (2011-2015)

\begin{tabular}{lcc}
\hline Diagnóstico tomográfico & $\mathrm{n}$ & $\%$ \\
\hline Dilatación ventricular & 9 & 23.8 \\
Dilatación ventricular-neoformación & 1 & 2.6 \\
Infarto cerebral-dilatación ventricular & 1 & 2.6 \\
$\quad$ Sub-total Dilatación ventricular y asociados & 11 & 29.0 \\
Neoformación encefálica & 5 & 13.2 \\
Edema-neoformación & 1 & 2.6 \\
$\quad$ Sub-total Neoformación y asociados & 6 & 15.8 \\
Hidrocefalia & 3 & 7.9 \\
Hidrocefalia-hipertensión endocraneana & 3 & 7.9 \\
$\quad$ Sub-total & 6 & 15.8 \\
Lesión isquémica & 2 & 5.3 \\
Lesión isquémica-contusión & 1 & 2.6 \\
Lesión isquémica-neoformación & 1 & 2.6 \\
$\quad$ Sub-total Lesiones isquémicas y asociadas & 4 & 10.5 \\
Hipertensión endocraneana & 2 & 5.3 \\
Hipertensión-colapso ventricular & 1 & 2.6 \\
$\quad$ Sub-total Hipertensión y asociados & 3 & 7.9 \\
Edema cerebral & 3 & 7.9 \\
Hemorragia-contusión & 2 & 5.3 \\
Calcificación dural tentorial & 1 & 2.6 \\
Colapso ventricular & 1 & 2.6 \\
Dilatación de cuernos temporales & 1 & 2.6 \\
$\quad$ Sub-total Otros & 8 & 21.0 \\
\hline Total & 38 & 100 \\
\hline & & \\
\hline
\end{tabular}

(7.9\%, 3/38) (Cuadro 2). En el Cuadro 3 se presentan los diagnósticos tomográficos más frecuentes y su asociación con las características demográficas más frecuentes de los pacientes. La Figura 1 muestra una imagen tomográfica típica de una severa dilatación ventricular compatible con hidrocefalia.

\section{Discusión}

En el presente estudio, la lesión encefálica más frecuente fue la dilatación ventricular (DV) con 11 casos, presentándo- se en nueve pacientes como lesión única y en dos casos asociados a infarto cerebral y neoformación encefálica. Es necesario reconocer cuando la ventriculomegalia es considerada como indicio patológico de enfermedades como la hidrocefalia o tumores cerebrales, por lo que es imprescindible la correlación de los hallazgos por imágenes con signos clínicos presentes en el paciente. Esto se debe a que la DV simétrica o asimétrica puede ser un hallazgo relativamente común en canes adultos y jóvenes normales (Vullo et al., 1997; Thomas, 1999). Además, cambios mínimos o leves en el tamaño y simetría 
Cuadro 3. Distribución de los diagnósticos tomográficos más frecuentes de lesiones encefálicas en canes, según las características demográficas más frecuentes (Lima, Perú, 2011-2015)

\begin{tabular}{|c|c|c|c|c|c|}
\hline \multirow{2}{*}{$\begin{array}{l}\text { Diagnóstico } \\
\text { tomográfico }\end{array}$} & \multicolumn{2}{|c|}{ Ocurrencia } & \multicolumn{3}{|c|}{ Característica más frecuente según: } \\
\hline & $\mathrm{n}$ & $\%$ & $\begin{array}{c}\text { Edad } \\
\text { (años) }\end{array}$ & Tamaño & Razas \\
\hline $\begin{array}{l}\text { Dilatación } \\
\text { ventricular }\end{array}$ & 11 & 29.0 & $1-7$ & Pequeño & Maltés, Poodle \\
\hline Neoformación & 6 & 15.8 & $>7$ & $\begin{array}{l}\text { Mediano y } \\
\text { grande }\end{array}$ & Labrador \\
\hline Hidrocefalia & 6 & 15.8 & $1-7$ & $\begin{array}{l}\text { Miniatura y } \\
\text { Pequeño }\end{array}$ & Chihuahua, Pug \\
\hline Lesión isquémica & 4 & 10.5 & $1-7$ & $\begin{array}{l}\text { Miniatura y } \\
\text { Pequeño }\end{array}$ & Yorkshire \\
\hline $\begin{array}{l}\text { Hipertensión } \\
\text { endocraneana }\end{array}$ & 3 & 7.9 & $1-7$ & Miniatura & Chihuahua \\
\hline Edema cerebral & 3 & 7.9 & $>7$ & $\begin{array}{l}\text { Mediano y } \\
\text { grande }\end{array}$ & $\begin{array}{c}\text { Cocker Spaniel, } \\
\text { Labrador }\end{array}$ \\
\hline Otros $^{1}$ & 5 & 13.1 & & & \\
\hline Total & 38 & 100 & & & \\
\hline
\end{tabular}

${ }^{1}$ Calcificación dural tentorial, colapso ventricular, dilatación de cuernos temporales, infarto cerebral, hemorragia cerebral, contusión cerebral
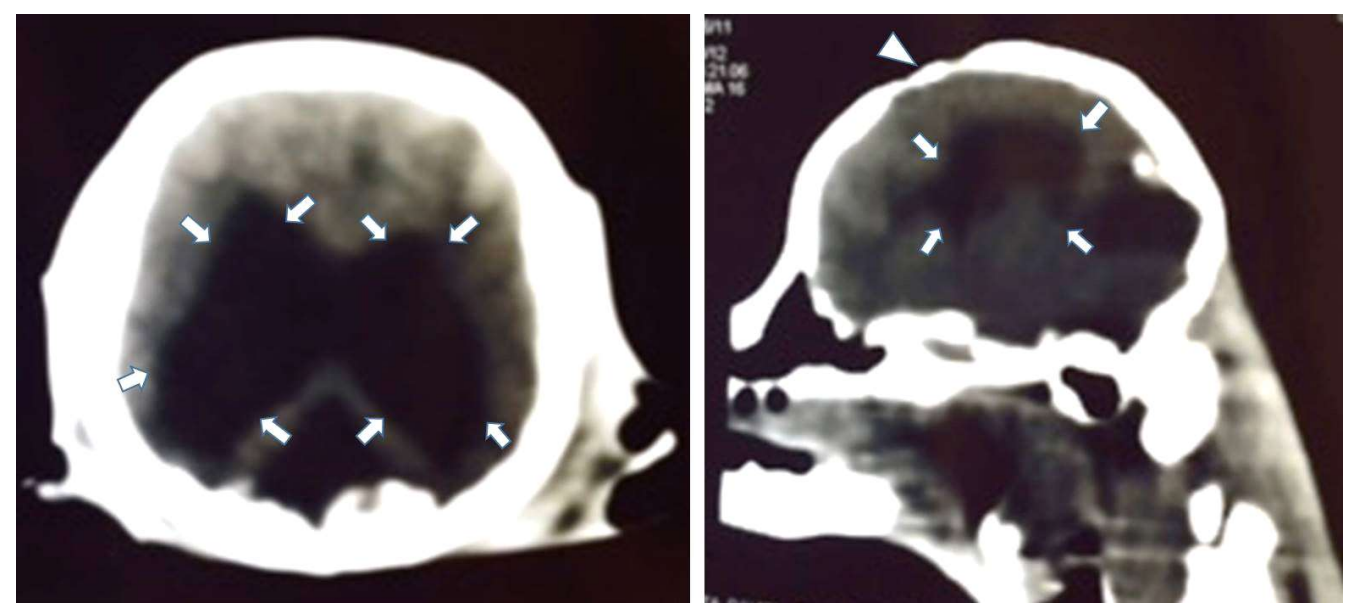

Figura 1. Tomografía computarizada cerebral en vista coronal (izquierda) y sagital (derecha) de una severa dilatación ventricular (delimitado por flechas blancas) compatible con hidrocefalia. Se identificó además fontanelas abiertas (cabeza de flecha) 
ventricular pueden estar relacionados a variables como raza, sexo o peso en perros clínicamente sanos (Vite et al., 1997; Woo et al., 2010).

Un caso de DV fue identificado asociado a infarto cerebral (IC). El IC se caracteriza por presentar un inicio agudo a subagudo, generando una progresión de signos neurológicos en un tiempo de 24 a 72 horas. El IC se produce por embolismos (neoplásicos, sépticos, colesterol) (Esparza et al., 2013) o trombos (problemas vasculares o plaquetarios) (Raurell et al., 2007; Babicsak et al., 2012; Esparza et al., 2013). Se han descrito tres fases en la evolución del infarto (aguda, subaguda y crónica), estando pobremente definido en las primeras horas de evolución, de allí que se requiere una segunda evaluación de TC pasadas las 72 horas. En su estado crónico (un mes por lo general) se inicia la reabsorción del tejido necrótico, lo que origina una retracción de la corteza cerebral adyacente al foco infartado. Esto finalmente producirá una $\mathrm{DV}$, aunque sin comunicación, debido a que la necrosis no afecta al epéndimo ni a la glía subependimaria (Echeneider y Liboman 1996).

Seis pacientes presentaron hidrocefalia (HI) (Figura 1), siendo tres de ellos con signos de hipertensión endocraneana. La HI constituía una enfermedad de rara identificación certera antes que las técnicas de imagenología no invasiva como la TC aparecieran en la práctica (Przyborowska et al., 2013). Estos avances lograron identificar a la HI como la causa de más de la mitad del total de casos de malformaciones congénitas cerebrales en canes (Carvalho et al., 2010). En pacientes jóvenes o menores de un año se distingue a la hidrocefalia de tipo congénito, donde los animales afectados cursan con diversidad de signos clínicos y variables grados de severidad (Orozco y Aranzazu, 2004). La TC tiene una buena capacidad de resolución para el diagnóstico de esta enfermedad, brindando una visualización de tres dimensiones del tamaño ventricular y reconocien- do con facilidad la presencia de líquido cefalorraquídeo (Thomas, 2010). La HI de carácter crónico ocurre generalmente en pacientes adultos a gerontes, caracterizándose por presentar una compensación relativa y asociada generalmente a neoplasias o a procesos inflamatorios crónicos (Orozco y Aranzazú, 2004).

Los casos de HI se identificaron mayormente en razas de tamaño miniatura y pequeña, lo cual coincide con otros autores (Orozco y Aranzazú 2004; Przyborowska et al., 2013). Las causas para su presentación pueden incluir anomalías en el desarrollo cerebral, infecciones durante el embarazo y hemorragias cerebrales (Esteve-Ratsch et al., 2001; Woo et al., 2010). Históricamente, las razas reportadas con predisposición a HI congénita son el Maltés, Yorkshire Terrier, English Bulldog, Chihuahua, Poodle miniatura, Pug, Pekinés, Pomeranian y Boston Terrier (Orozco y Aranzazú, 2004; Thomas, 2010), siendo en el presente trabajo las razas Chihuahua, Pug, Maltés y Poodle.

Se diagnosticaron cinco casos de neoformaciones cerebrales (NC), de los cuales uno estuvo asociado a edema cerebral. $\mathrm{Su}$ presencia estuvo relacionada a procesos neoplásicos de tipo cancerígeno. Estos resultados concuerdan con Babicsak et al. (2011), quiénes reportan la capacidad de la TC en la identificación de tumores cerebrales e incluso en sugerir determinado tipo de presentación tumoral. La mayor frecuencia de $\mathrm{NC}$ se encontró en canes gerontes ( $>7$ años), lo cual coincide con Snyder et al., 2006), quienes mencionan que los tumores intracraneales se diagnostican principalmente en animales mayores de ocho años, donde el riesgo de presentar tumores primarios intracraneales incrementa en un $40 \%$ cada año de vida. Así mismo, Park (2003) señala que los tumores encefálicos son diagnosticados usualmente en canes de mediana a mayor edad con tendencia a localizarse en la sustancia blanca y alrededor del cuarto ventrículo. 
Se diagnosticaron tres pacientes con edema cerebral de tipo difuso y un caso de tipo vasogénico asociado a neoformación encefálica. Cabe indicar que el motivo de someter a los canes a TC en los primeros casos fue a consecuencia de politraumatismos encéfalo craneano. El hallazgo tomográfico principal fue de un cerebro hipodenso, con pérdida de diferenciación entre la sustancia gris y blanca, así como el aumento del volumen de los hemisferios cerebrales afectados. Así mismo, se pudo identificar compresión ventricular y borramiento del patrón de surcos y circunvoluciones. Uscanga et al. (2005) indican que la TC permite identificar al edema cerebral difuso aun en estadios tempranos del trauma. Esto puede indicar además un valor predictivo de la TC, convirtiéndose en una herramienta valiosa de decisión al momento de instaurar el tratamiento para evitar la evolución fatal del paciente politraumatizado.

En el presente trabajo se presentaron canes sin alteraciones en el diagnóstico tomográfico, lo cual podría relacionarse con algunas limitaciones de la TC en la evaluación del encéfalo, como la baja calidad de contraste en tejidos blandos, lo que puede reducir la información para la mayoría de las enfermedades intracraneales. Asimismo, la captación de la imagen de la fosa caudal (cerebelo y tronco encefálico) puede ser problemática debido al artefacto de endurecimiento del haz (Dennis, 2003). Es recomendable hacer hincapié en el estudio de estas limitaciones en trabajos futuros.

La meta médica es la aproximación al diagnóstico temprano e identificación de la causa de la dolencia para instaurar de forma temprana el tratamiento adecuado. En ese sentido, la TC aporta su capacidad de información tridimensional, diferenciación de tejidos blandos adyacentes y fina resolución en la evaluación encefálica. Estas ventajas la convierten en una modalidad adecuada para la evaluación encefálica en los pacientes caninos, ya que, generalmente, las estructuras cráneo encefálicas son complicadas de eva- luar por los métodos radiográficos convencionales.

\section{ConClusiones}

- Se identificaron 38 diagnósticos tomográficos positivos a lesión encefálica en una población de 71 pacientes sometidos a tomografía computarizada encefálica, representando el $53.5 \%$ de los pacientes.

- Las lesiones diagnosticadas por tomografía computarizada en canes más frecuentes fueron dilatación ventricular (29\%), neoformación encefálica (15.8\%) e hidrocefalia $(15.8 \%)$.

\section{Literatura Citada}

1. Aguinaga HF, Rivera JA, Tamayo LJ, Tobón M, Osorno RC. 2006. Tomografía axial computarizada y resonancia magnética para la elaboración de un atlas de anatomía segmentaria a partir de criosecciones axiales del perro. Rev Colomb Cienc Pec 19: 451-459.

2. Babicsak V, Machado V, dos Santos D, Zardo K, Campos L, Vulcano L. 2012. Aspectos de infartos hemorrágicos em cães na tomografia computadorizada e ressonância magnética. Vet Zootec 19: 66-72.

3. Babicsak V, Zardo K, dos Santos D, Carandina L, Machado V, Vulcano L. 2011. Aspectos tomográficos de tumores cerebrales primarios en perros y gatos. Vet Zootec 18: 531-541.

4. Calzado A, Gelejins J. 2010. Tomografía computarizada. Evolución, principios técnicos y aplicación. Rev Fis Med 11: 163-180.

5. Carvalho CF, Chammas MC, Andrade JP, Jimenez CD, Diniz SA, Cerri GG. 2010. Transcranial duplex ultrasonography in dogs with hydrocephalus. Arq Bras Med Vet Zoo 61: 54-63. doi: 10.1590/S0102-09352010000100008 
6. Dennis R. 2003. Advanced imaging: indications for $\mathrm{CT}$ and MRI in veterinary patients. In Practice 25: 243-245. doi: 10.1136/inpract.25.5.243

7. Schneider LB, Libman RB, Kanner R. 1996. Utility of repeat brain imaging in stroke. Am J Neuroradiol 17: 1259-1263.

8. Esparza E, Rodríguez D, Benavidez E. 2013. Síndrome vestibular por accidente cerebrovascular asociado a hipotiroidismo secundario. Spei Domus 9: 57-63. 10.16925/sp.v9i19.710

9. Esteve-Ratsch B, Kneissl S, Gabler C. 2001. Comparative evaluation of the ventricles in the Yorkshire Terrier and the German Shepherd dog using low-field MRI. Vet Radiol Ultrasoun 42: 410-413. doi: 10.1111/j.1740-8261.2001.tb00960.x

10. Lorigados C, Pinto, A. 2013. La tomografía computarizada del cerebro del perro: aspectos de correlación anatómica normal. Arq Bras Med Vet Zoo 65: 729-734.

11. Luján A. 2007. Trauma craneal II: tratamiento y pronóstico. Clin Vet Peq Anim 27: 173-177.

12. Machado T, Ferrigno C, Stopiglia A. 2007. Revisión anatómica del seno venoso sagital dorsal en canes braquicéfalos. Pesqui Vet Brasil 27: 194-198.

13. Orozco S, Aranzazú D. 2004. Diagnóstico clínico patológico de tres casos de hidrocefalia congénita en perros. Vet México 35: 379-386.

14. Park CH. 2003. Oligodendroglioma in a French Bulldog. J Vet Sci 4: 195-197.

15. Przyborowska P, Adamiak $Z$, Jaskolska M, Zhalniarovich Y. 2013. Hidrocephalus in dogs: a review. Vet Med-Czech 58: 73-80

16. Raurell X, Centellas C, Zamora A. 2007. Enfermedad vascular cerebral: 9 casos clínicos. Clín Vet Peq Anim 27: 27-35.
17. Rodríguez M, Dosouto V, Rosales Y, Musle M, González Y. 2010. Valor de la tomografía axial computarizada para el diagnóstico precoz del traumatismo craneoencefálico. Medisan 14: 767-773.

18. Snyder JM, Shofer FS, Van Winkle TJ, Massicotte C. 2006. Canine intracranial primary neoplasia: $173 \mathrm{ca}-$ ses (1986-2003). J Vet Intern Med 20: 669-675. doi: 10.1111/j.1939-1676.2006.tb02913.x

19. Thomas WB. 1999. Nonneoplastic disorders of the brain. Clin Tech Small An P 14: 125-147. doi: 10.1016/S10962867(99)80030-9

20. Thomas WB. 2010. Hydrocephalus in dogs and cats. Vet Clin N Am-Small 40: 143-159. doi: 10.1016/j.cvsm.2009.09.008

21. Uscanga MC, Castillo JA, Arroyo G. 2005. Hallazgos por tomografía computada en pacientes con trauma craneoencefálico, su relación con la evolución clínica y cálculo del edema cerebral. Rev Neurol Neurocir Psiquiat 38: 11-19.

22. Vite CH, Insko EK, Schotland HE, Panckeri K, Hendricks JC. 1997. Quantification of cerebral ventricular volume in English Bulldogs. Vet Radiol Ultrasoun 38: 437-443. doi: 10.1111/ j.1740-8261.1997.tb00868.x

23. Vullo T, Manzo R, Gomez DG, Deck MD, Cahill PT. 1997. Diagnosis of cerebral ventriculomegaly in normal adult Beagles using quantitative MRI. Vet Radiol Ultrasound 38: 277-281. doi: 10.1111/j.1740-8261.1997.tb00855.x

24. Woo DC, Choi CB, Nam JW, Ryu KN, Jahng GH, Lee SH, Lee DW, Kim HY, Kim HY, Ahn KJ, Choe BY. 2010. Quantitative analysis of hydrocephalic ventricular alterations in Yorkshire terriers using magnetic resonance imaging. Vet Med-Czech 55: 125-132. 